\title{
Performance Studies of an Optical-Fiber OSL/RL Dosimetry System in Pulsed High-Intensity Radiation Beams
}

\author{
David Benoit ${ }^{\mathrm{a}, \mathrm{b}}$, Laurent Dusseau ${ }^{\mathrm{a}}$, Maurice Glaser ${ }^{\mathrm{c}}$, Bhaskar Mukherjee ${ }^{\mathrm{d}}$, Federico Ravotti ${ }^{\mathrm{c}, 1}$ \\ a'IES-Institut d'Electronique du Sud, Université Montpellier II, 34095 Montpellier, France \\ ${ }^{\mathrm{b}}$ Département de Radiothérapie, Centre Léon Bérard, 69008 Lyon, France \\ ${ }^{\circ}$ CERN Physics Department, 1211 Geneva 23, Switzerland \\ ${ }^{\mathrm{d} D E S Y}$ MSK Group, 22607 Hamburg, Germany
}

\begin{abstract}
The SrS:Ce,Sm phosphor is suitable for operation in harsh radiation environments where real-time dosimetry measurements are needed to survey the Total Ionizing Dose (TID) damage in electronic components. For these applications, the OSL and RL emissions from this phosphor can be exploited by mounting the SrS crystal at the edge of a single, radiation hard, optical fiber. In this work we present an exhaustive characterization of this real-time OSL/RL probe including stability, repeatability of the measured signals, dosimetry performance and measurements of the temporal behavior of the RL signal in pulsed particle beams.
\end{abstract}

\section{Introduction}

Monitoring ionizing radiation around High Energy Physics (HEP) beams is a difficult task due to the complexity and intensity of the generated radiation fields. The phosphor SrS:Ce,Sm developed by the University Montpellier II in France proved its suitability for operation in harsh radiation environments where dose measurements are needed to survey the Total Ionizing Dose (TID) damage in electronic components (Ravotti et al., 2004). Both Optically Stimulated Luminescence (OSL) and RadioLuminescence (RL) emissions from the SrS:Ce,Sm can be exploited for such a purpose (Ravotti, et al., 2007).

In this work we investigated the performance of a real-time monitoring system based on a SrS crystal mounted at the edge of a single, large core, optical fiber. With this probe layout, the OSL and RL luminescent signals are guided away from the measurement point, collected at the other end of the fiber by means of a photomultiplier tube (PMT), and processed by a dedicated DAQ system (Benoit et al., 2008). For HEP applications, particular care has been taken in the manufacturing of this monitoring probe in terms of geometry, dimensions, stimulation-collection efficiency and radiation hardness. In particular, the chosen optical fiber is a custom made radiation-hard quartz fiber from Polymicro Technologies, USA, of 600 $\mu$ m core diameter (Fernandez Fernandez et al., 2008). The probe head consists of a SrS sample embedded in a Hostaform ${ }^{\circledR}(\mathrm{POM})$ or a Dural $^{\circledR}$ cylinder of $3 \mathrm{~mm}$ diameter and $10 \mathrm{~mm}$ length. The choice of the head material is made according to the composition of the radiation field to monitor.

Radiation damage survey in HEP is usually performed with semiconductor devices that allow for measuring the TID with an accuracy of $\pm 20 \%$ in harsh radiation fields (Ravotti, et al., 2007b). The aim of this work is to qualify this OSL/RL probe as a

\footnotetext{
${ }^{1}$ Corresponding Author: Federico Ravotti, CERN, 1211, Geneva 23, Switzerland, Tel. +41-76-4875643, e-mail: Federico.Ravotti@cern.ch.
} 
complementary tool for routine monitoring in HEP. For this purpose, stability and repeatability of the measured OSL/RL signals have been investigated, together with the accuracy of the TID measurement in OSL mode against other dosimetry means commonly used in HEP. A measurement depicting the temporal behavior of the RL signal is also presented here, demonstrating that this radiation monitor can be simultaneously used as an on-line time-structure monitor for pulsed particle beams.

\section{Irradiation Experiments}

The irradiation campaigns were performed in two facilities where radiation fields, representative of the environmental conditions found in HEP, are available.

At the FLASH facility of DESY, the field was composed of electrons and photons (Mukherjee and Simrock, 2008). During routine operation at FLASH, copious levels of bremsstrahlung photons are produced due to the collision of electrons with the accelerator parts; in particular, an intense and continuous radiation field is expected at the RF Gun region of the accelerator, where our monitoring system was tested. The measurement probe used at FLASH had a sensitive head encapsulated in POM plastic. In the DESY experiment, the stability of the OSL signal has been measured over a long period of stable RF power. A laser pulse of $1.5 \mathrm{~s}$ duration was used to stimulate the SrS phosphor and the peak amplitude of the resulting OSL signal decay was recorded.

In the IRRAD6 facility at CERN Proto-Synchrotron (PS) complex (Glaser et al., 2006), a secondary radiation field composed mainly of neutrons, photons and charged hadrons is generated from the interaction of the primary $24 \mathrm{GeV} / \mathrm{c}$ protons with a beam absorber. In this mixed radiation field the dose-rates and the particle intensities are close to the one expected in hadron facilities like the CERN Large Hadron Collider. In normal operation, the proton bursts are delivered from the CERN PS accelerator in "spills" of about $350 \mathrm{~ms}$ width and with an average intensity of $\sim 2-4 \times 10^{11} \mathrm{p} /$ spill. The probe in IRRAD6 was placed $27 \mathrm{~cm}$ off beam axis in the radial direction and $10 \mathrm{~cm}$ from the absorber along the beam line. To reduce the sensitivity to the fast neutrons component of IRRAD6, the used probe had a sensitive head encapsulated in a Dural ${ }^{\circledR}$ alloy. In the CERN experiment both RL and OSL signals were measured. The RL signal was directly recorded from the PMT by means of an oscilloscope while the OSL decays was triggered by a 2 s laser stimulation.

In OSL mode, both the DESY and the CERN probe were initially calibrated with gamma-rays showing a linear sensitivity up to $150 \mathrm{mV} / \mathrm{cGy}$ (Benoit et al., 2008).

\section{Results and Discussion}

\subsection{Stability in $O S L$}

The stability measurement performed at the FLASH facility is shown in Fig. 1. Here, the cumulated dose measured via OSL every 15min (expressed in the plot in terms of mean dose-rate), is compared with the temporal behavior of the RF power as function of the experiment time. The dose integration time (15min) was chosen to obtain a satisfying signal-to-noise ratio. The OSL measurements match well the behavior of the RF Gun power and the recorded dose-rate is consistent with early dose measurements performed with TLDs (Mukherjee and Simrock, 2008). The stability of the OSL signal can be evaluated during the $10 \mathrm{~h}$ period of stable RF power. All OSL measurements spread in a range of $\pm 5 \%$ with respect to their mean value. Only one point presents a variation to the mean of more than $10 \%$.

\subsection{Repeatability in $O S L$ and $R L$}

The repeatability is defined as the ratio of the standard deviation over the measurements mean. In OSL, the repeatability has been evaluated in IRRAD6 by using a set of 229 measurements recorded with a dose integration time of 1.66min. This time 
interval has been chosen to allow the integration of a TID produced by 12 consecutive proton spills (a few Gy) delivered to the beam absorber. The number of incident protons per spill varied at each extraction and it was precisely measured by means of a Secondary Emission Chamber (Bernier et al., 1997). The result of this measurement, shown in Fig. 2, indicates a repeatability of about $6 \%$.

For the RL, the repeatability has been evaluated using a set of 15 measurements by integrating 12 consecutive proton spills each. The integral of the RL signal has been directly measured with the oscilloscope. The repeatability in this operation mode has been found to be better than $2 \%$, confirming previous measurements carried out with the same probe in a photon field (Benoit et al., 2008b).

\subsection{Accuracy of the dose measurement in OSL}

In order to compare the results of our system with other dosimetry methods used in routine at CERN, different passive dosimeters (Radio-Photo Luminescent -RPL- and Gafchromic films HD-810 (Glaser et al., 2006)) were exposed during 15h together with the sensitive head. Three to four samples of each dosimeter type were simultaneously irradiated.

The results obtained are summarized in Table 1 . The dosimetry measurement carried out with our system in OSL, by adding the dose recorded during several measurements over the $15 \mathrm{~h}$ exposure time, is in agreement within $20 \%$ with respect to the RPL measurement. The RPL has been chosen as reference because of its reduced sensitivity for the IRRAD6 neutrons (Ravotti, et al., 2007b). This result is promising when taking into account the complexity of the radiation field and the different calibration conditions of each dosimeter. The different calibration conditions are also accounted in the computation of the experimental error that affects the values presented in Table 1.

\subsection{Temporal behaviour of the RL signal}

To investigate the capability of the system to temporally resolve the particle beam's structure concurrently with dose measurement, Fig. 3 (top) shows an RL measurement of a $\sim 350 \mathrm{~ms}$ wide proton spill in IRRAD6 directly recorded with the oscilloscope at the output of the PMT. Although the probe in IRRAD6 was not placed directly in the primary beam direction, the signal measured by the SrS phosphor, clearly maintains the original time structure of the incoming particle beam. Despite the noise that affects this measurement, the temporal structure of the proton spill is clearly visible and in good agreement with the one monitored in the PS accelerator with a dedicated device (air scintillation chamber shown in the bottom of Fig. 3 (Johnson et al., 1976)). Repeated measurements showed similar agreement, and also revealed the possibility to discriminate faster proton spills down to $40 \mathrm{~ns}$ time-width and spaced $265 \mathrm{~ns}$ apart.

\section{Conclusion}

In this work we characterized a real-time optical fiber OSL/RL dosimetry system based on the SrS:Ce,Sm phosphor for its routine utilization in HEP. The peculiarity of HEP radiation environment (mixed and intense fields) requires a detailed characterization of the sensors that have to be used in it. The performance of our system in the bremsstrahlung photons of DESY and in the mixed hadron field of CERN lie well within the $20 \%$ precision limit required in HEP dosimetry applications for radiation damage survey. In addition to this, the analysis of the time behavior of the RL signal emitted by the SrS:Ce,Sm, promotes the use of this OSL/RL system as a multi-purpose, real-time beam monitor device for fast and intense HEP particle beams. 


\section{References}

Benoit, D., Garcia, P., Matias-Vaillé, S., Ravotti, F., Vaillé, J.-R., Glaser, M., Brichard, B., Fernandez Fernandez, A., Chatry, C., Dusseau, L., 2008. Real-time fibered optically stimulated luminescence dosimeter based on SrS:Ce,Sm phosphor. IEEE Trans. Nucl. Sci., 55(4), 2154-2160.

Benoit, D., Vaillé, J.-R., Lautissier, J., Matias-Vaillé, S., Isturiz, J., Garcia, P., Brichard, B., Dusseau L., 2008b. Feasibility of Fibered Monitoring System for Pulsed Dose-Rate Facilities Based on Radioluminescence of SrS:Ce,Sm Phosphor. IEEE Trans. Nucl. Sci., 55(6), 3421-3427.

Bernier, K., Ferioli, G., Hatziangeli, E., Marchionni, A., Palladino, V., Stevenson, G.R., Tsesmelis, E., de Rijk, G., 1997. Calibration of secondary emission monitors of absolute proton beam intensity in the CERN SPS North Area. CERN Yellow Report 97-07.

Fernandez Fernandez, A., Brichard, B., O'Keeffe, S., Fitzpatrick, C., Lewis, E., Vaillé, J.-R., Dusseau, L., Jackson, D.A., Ravotti, F., Glaser, M., El-Rabii, H., 2008. Real-time fiber optic radiation dosimeters for nuclear environment monitoring around thermonuclear reactors. Fus. Eng. Des., 83(1), 50-59.

Glaser, M., Ravotti, F., Moll, M., 2006. Dosimetry Assessments in the Irradiation Facilities at the CERN-PS Accelerator. IEEE Trans. Nucl. Sci., 53(4), 2016-2022.

Johnson, C., Actis, P., Dorenbos T., 1976. An optical coupled differential beam time structure monitor for slow extraction. CERN Report PC/CCI/Note 76-7.

Mukherjee, B., and Simrock, S., 2008. Accelerator dosimetry at free electron lasers in Hamburg. Rad. Meas., 43, 1154-1159.

Ravotti, F., Glaser, M., Moll, M., Idri, K., Vaillé, J.-R., Prevost, H., Dusseau, L., 2004. Conception of an integrated sensor for the radiation monitoring of the CMS experiment at the Large Hadron Collider. IEEE Trans. Nucl. Sci., 51(6), 3642-3648.

Ravotti, F., Benoit, D., Lefebvre, P., Valvin, P., Vaillé, J.-R., Dusseau, L., Fesquet, J., Gasiot, J., 2007. Time-resolved photoluminescence and optically stimulated luminescence measurements of picosecond-excited SrS:Ce,Sm phosphor. Journal of Applied Physics, 102(12), article 123102, 5 pages.

Ravotti, F., Glaser, M., Rosenfeld, A.B., Lerch, M.L.F., Holmes-Siedle, A.G., Sarrabayrouse, G., 2007b. Radiation Monitoring in Mixed Environments at CERN: from the IRRAD6 Facility to the LHC Experiments. IEEE Trans. Nucl. Sci., 54(4), 1170-1177. 


\section{Captions}

Table 1: Accuracy of the OSL dose measurement at the CERN IRRAD6 facility.

Fig 1: Stability of the OSL measurements at the DESY FLASH facility. The dose-rate measured in OSL is compared with the RF power of the accelerator as function of the irradiation time.

Fig. 2: Repeatability of the OSL measurements in IRRAD6. The measured OSL signal in Volts is plotted as function as the measurements number ( $N=229)$. The dashed lines represent $\pm 1 \sigma$ with respect to the mean value (straight line).

Fig 3: RL measurement of a proton spill at CERN IRRAD6. The RL signal from the PMT measured with the oscilloscope (top) is compared with the same measurement performed with the PS beam instrumentation (bottom). 


\begin{tabular}{|c|c|c|}
\hline Dosimeter & $\begin{array}{c}\text { Absorbed dose } \\
(\mathbf{G y})\end{array}$ & $\begin{array}{c}\text { Variation }^{(\mathbf{1})} \\
(\boldsymbol{\%})\end{array}$ \\
\hline RPL & $67.3( \pm 7 \%)$ & 0.0 \\
\hline OSL & $53.6( \pm 6 \%)$ & -20.4 \\
\hline Gaf. Film HD-810 & $78.4( \pm 7 \%)$ & +16.5 \\
\hline
\end{tabular}

(1) The variation is calculated as: $\left(\mathrm{D}_{\mathrm{x}}-\mathrm{D}_{\mathrm{RPL}}\right) / \mathrm{D}_{\mathrm{RPL}} * 100$. 


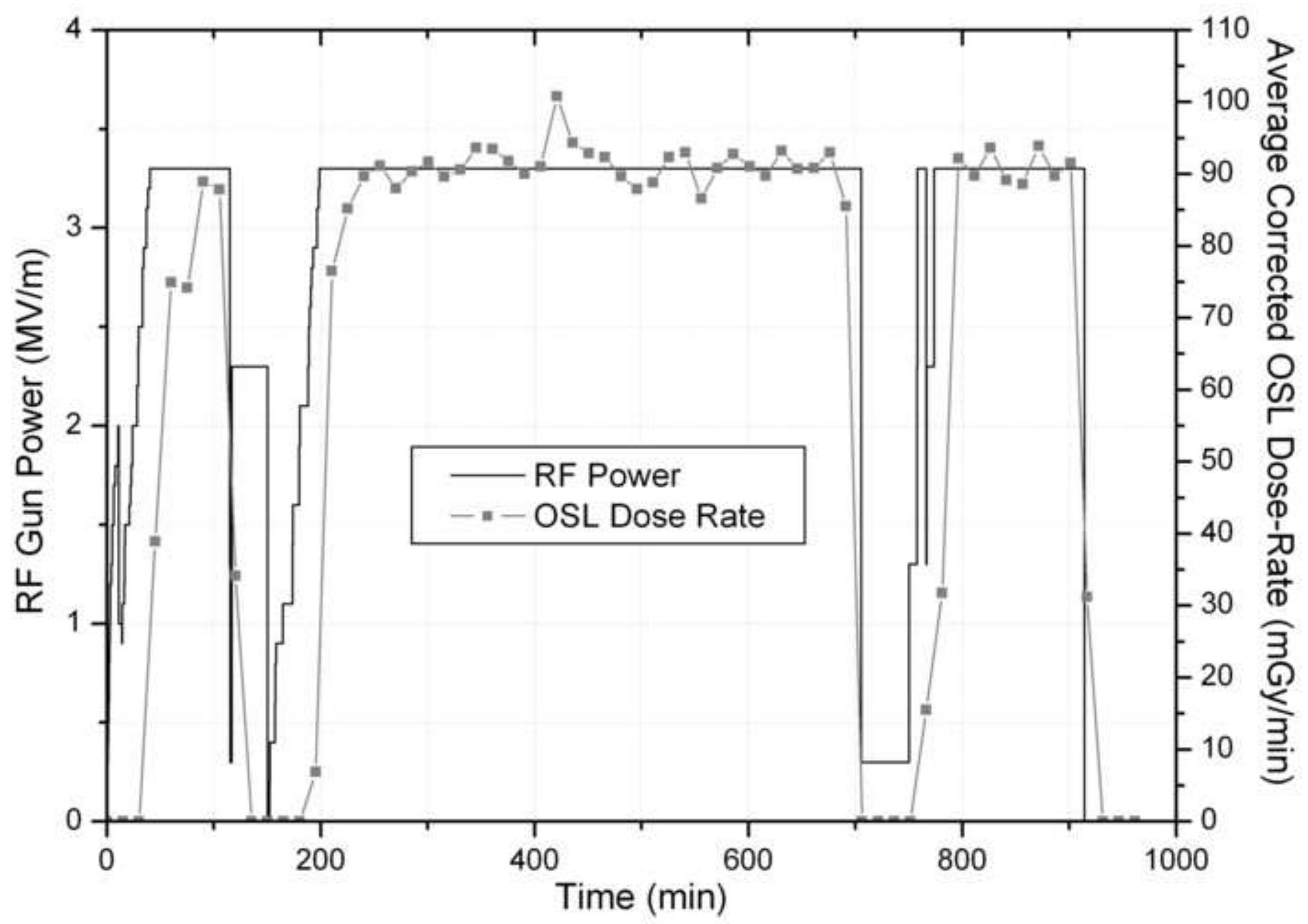




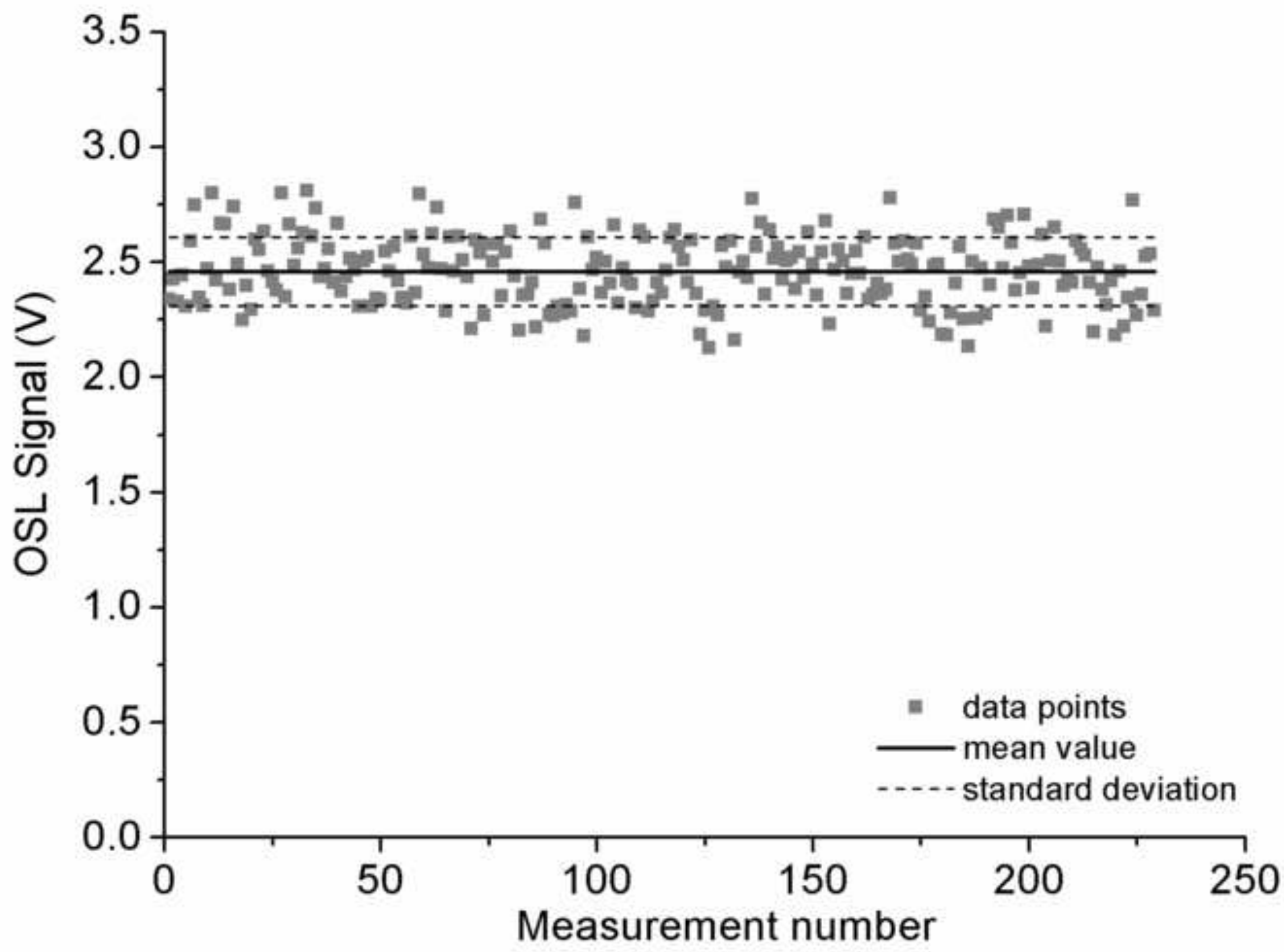


